\title{
Robot-assisted laparoscopic prostatectomy performed in a patient with severe thrombocytopenia due to primary myelofibrosis
}

\author{
Rose Khavari, MD; Lambros Stamatakis, MD; Brian Miles, MD
}

\begin{abstract}
Robot-assisted laparoscopic prostatectomy (RALP) has emerged as a minimally invasive alternative to open radical prostatectomy (ORP) for the treatment of clinically localized prostate cancer. In comparison to the open procedure, there is significantly less intraoperative blood loss during RALP. This benefit has allowed RALP to become a feasible option for patients who would be poor candidates for ORP, including those patients with intrinsic hematological disorders. In this case study, we report a successfully performed RALP in a patient with severe thrombocytopenia in the presence of primary myelofibrosis.
\end{abstract}

Can Urol Assoc J 2009;3(4):E23-E24

\section{Case report}

A 55-year-old asymptomatic man with an elevated serum prostate-specific antigen level of $4.9 \mu \mathrm{g} / \mathrm{L}$ and a normal digital rectal examination underwent a transrectal ultrasonographyguided biopsy of his prostate and was diagnosed with adenocarcinoma of the prostate (Gleason $3+3$ ). His medical history was significant for primary myelofibrosis, as well as type 2 diabetes mellitus. He was previously diagnosed with primary myelofibrosis by bone marrow biopsy, and genomic studies demonstrated that he had a point mutation in the JAK2 gene. All risks of the surgery with particular emphasis on bleeding, and all other options including radiotherapy, watchful waiting and cryosurgery were discussed in depth with the patient. The patient and his family chose to proceed with radical prostatectomy. Preoperative laboratory studies demonstrated a hemoglobin concentration of $106 \mathrm{~g} / \mathrm{L}$ and a platelet count of $22 \times 10^{9}$ cells/L.

The patient was admitted on the evening before surgery and, in conjunction with the recommendations of the hematology service, he was transfused $600 \mathrm{~mL}$ of leukoreduced platelets and received standard dosing of intravenous aminocaproic acid every 6 hours. On the morning of surgery, his platelet count had modestly increased to $28 \times 10^{9}$ cells $/ \mathrm{L}$. An additional $600 \mathrm{~mL}$ of platelets were administered, which increased his platelet count to
$42 \times 10^{9}$ cells/L just before entering the operating room. The patient then underwent an uneventful robot-assisted laparoscopic prostatectomy (RALP), as well as restoration of the posterior aspect of the rhabdosphincter and the puboprostatic collar, as described by Rocco and colleauges ${ }^{1}$ and Tewari and coauthors, ${ }^{2}$ respectively. About $8 \mathrm{~mL}$ of fibrin sealant (Evicel, Ethicon, Inc.) was generously applied to the surgical bed and a Jackson-Pratt drain was placed near the urethrovesical anastomosis. The total operative time was 197 minutes, and the estimated blood loss was $250 \mathrm{~mL}$. Of note, the patient remained hemodynamically stable throughout the procedure, but was transfused an additional $600 \mathrm{~mL}$ of platelets during the procedure to help maintain his platelet concentration. His immediate postoperative complete blood count revealed a hemoglobin and platelet concentration of $87 \mathrm{~g} / \mathrm{L}$ and $55 \times 10^{9}$ cells/L, respectively. He was admitted to the surgical intensive care unit on the evening of surgery for close monitoring. He remained hemodynamically stable and there was minimal drainage from the Jackson-Pratt drain. The patient was transferred to the recovery ward on the first postoperative day and discharged home 24 hours later after the return of his bowel function and removal of the drain. On the day of discharge, his hemoglobin concentration was $85 \mathrm{~g} / \mathrm{L}$ and his platelet count was $30 \times 10^{9}$ cells/L.

\section{Discussion}

Prostate cancer is the most common solid organ malignancy in men in the United States and about 186000 men will be diagnosed with prostate cancer in 2008. ${ }^{3}$ Localized prostate cancer can be treated by radical prostatectomy, which involves complete removal of the prostate and seminal vesicles. Although it provides excellent cancer control, open radical prostatectomy (ORP) can result in excessive intraoperative blood loss. One single-surgeon series of 1000 consecutive patients undergoing anatomical retropubic radical prostatectomy demonstrated an average blood loss of $818 \mathrm{~mL} .{ }^{4}$ Preoperatively, patients are often encouraged to undergo autologous blood donation because of the frequent 
Khavari et al.

need for intraoperative or postoperative blood transfusion. Some groups have also advocated preoperative administration of erythropoietin to help increase red blood cell mass before the procedure. ${ }^{5}$

RALP results in significantly less intraoperative blood loss than ORP. In a prospective single-surgeon series of 279 patients, the average intraoperative blood loss during RALP was $191 \mathrm{~mL}$, versus $664 \mathrm{~mL}$ during ORP $(p<0.001) .{ }^{6}$ A recent meta-analysis evaluating the outcomes of 19 studies also demonstrated that patients undergoing RALP experienced significantly less intraoperative blood loss compared with patients undergoing ORP. Moreover, patients undergoing RALP were $77 \%$ less likely to receive perioperative blood transfusion than their counterparts undergoing ORP.?

Other options for treatment of clinically localized prostate cancer include external beam radiotherapy and seed implant brachytherapy. Radiation-induced complications can include rectal bleeding and hematuria, and can be prolonged in up to $5 \%-10 \%$ of patients. ${ }^{8}$ We believed that if our patient were to develop these complications in the setting of his chronic thrombocytopenia, the bleeding could have become difficult to control and led to unacceptable morbidity. The patient and his family were extensively counseled regarding other available options for localized prostate cancer, with emphasis on the risk of severe bleeding during surgery, and the patient and his family chose to proceed with RALP.

Severe thrombocytopenia is a relative contraindication for surgery. In patients undergoing most elective surgical procedures, it is recommended that a platelet concentration of at least $50 \times 10^{9}$ cells $/ \mathrm{L}$ be maintained during the procedure. ${ }^{9}$ In conjunction with the hematology and anesthesiology services, we were able to prepare the patient appropriately in the perioperative setting and optimize his hematological parameters.

\section{Conclusion}

RALP can be performed safely in patients with severe throm- bocytopenia in the setting of intrinsic hematological disorders. Although this approach can be feasible, there is a significant risk of bleeding. Therefore, all other options for the treatment of localized prostate cancer need to be thoroughly discussed with the patient and family, and the risk of severe hemorrhage emphasized while obtaining the informed consent. Coordination with hematology and anesthesiology services can provide an optimal setting for the safe performance of RALP in such patients.

From the Scott Department of Urology, Baylor College of Medicine, Houston, Tex.

This article has been peer reviewed.

Competing interests: None declared.

\section{References}

1. Rocco B, Carmignani L, Acquati P, et al. Early continence recovery after open radical prostatectomy with restoration of the posterior aspect of the rhabdosphincter. Eur Urol 2007;51:996-1003.

2. Tewari AK, Bigelow K, Rao S, et al. Anatomic restoration technique of the continence mechanism and preservation of the puboprostatic collar: a novel modification to achieve early urinary continence in men undergoing robotic prostatectomy. Urology 2007;69:726-31.

3. Surveillance epidemilogy and end results. Bethesda (MD): National Cancer Institute. Available: http://seer.cancer.gov/statistics/ (accessed 2009 July 14).

4. Lepor H, Nieder AM, Ferrandino MN. Intraoperative and postoperative complications of radical retropubic prostatectomy in a consecutive series of 1000 cases. J Urol 2001;166:1729-33.

5. Nieder AM, Rosenblum N, Lepor H. A comparison of two different doses of preoperative recombinant erythropoietin in men undergoing radical retropubic prostatectomy. Urology 2001;57:737-41.

6. Farnham SB, Webster TM, Herrel SD, et al. Intraoperative blood loss and transfusion requirements for robotic-assisted radical prostatectomy versus radical retropubic prostatectomy. Urology 2006; 67:360-3.

7. Parsons JK, Bennett JL. Outcomes of retropubic, laparoscopic, and robotic-assisted prostatectomy. Urology 2008;72:412-6.

8. Catalona WJ, Misop H. Definitive therapy for localized prostate cancer - an overview. In: Wein AJ, Kavoussi LR, Nowik AC, editors. Campbell-Walsh urology. 9th ed. Philadelphia: Saunders Elseviers; 2007. p. 2939.

9. Wall MH, Prielipp RC. Transfusion in the operating room and the intensive care unit: current practice and future directions. Int Anesthesiol Clin 2000;38:149-69.

Correspondence: Dr. Rose Khavari, Scott Department of Urology, Baylor College of Medicine, 6620 Main St., Houston TX 77030; khavari@bcm.tmc.edu 